\title{
Globe
}

Revue internationale d'études québécoises

\section{Étranger et territorialité. D'une approche pluridisciplinaire à une approche transdisciplinaire}

\section{Carmen Mata Barreiro}

Volume 10, numéro 1, 2007

Étranger et territorialité

URI : https://id.erudit.org/iderudit/1000076ar

DOI : https://doi.org/10.7202/1000076ar

Aller au sommaire du numéro

Éditeur(s)

Globe, Revue internationale d'études québécoises

ISSN

1481-5869 (imprimé)

1923-8231 (numérique)

Découvrir la revue

Citer ce document

Mata Barreiro, C. (2007). Étranger et territorialité. D’une approche pluridisciplinaire à une approche transdisciplinaire. Globe, 10(1), 15-29.

https://doi.org/10.7202/1000076ar d'utilisation que vous pouvez consulter en ligne. 


\section{INTRODUCTION.}

\section{ÉTRANGER ET TERRITORIALITÉ. \\ D'UNE APPROCHE}

PLURIDISCIPLINAIRE À UNE

APPROCHE TRANSDISCIPLINAIRE

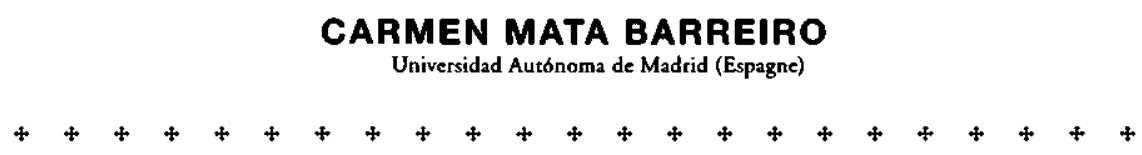

Dans le débat social et le domaine politique actuels, ainsi que dans l'univers de la recherche, le rapport entre l'être humain et l'espace - qui, devenu une dimension de l'existence humaine, se métamorphose en territoire - constitue un thème-clé. L'engouement pour la ville, les nouveaux territoires urbains nés de la suburbanisation, la périurbanisation et la métropolisation ${ }^{1}$, les "mégalopoles" ${ }^{2}$ et les «métapoles ${ }^{3}$ ", ou la valorisation de notions telles qu' "estuaires" ", associées à des cours d'eau, de même que l'importance accordée à la mémoire des lieux - et à des «lieux de mémoire" " - déterminent la mise en œuvre de projets et de recherches basés sur l'articulation de la mémoire, de l'identité et du territoire.

En interaction avec le territoire, le voyage, la mobilité et l'immigration mettent en scène l'étranger, qu'il soit voyageur, touriste ou immigrant. Le territoire est ainsi perçu comme un espace délimité par des

$$
+4
$$

1. Voir, par exemple, Hélène RIVERE D'ARC [éd.], Nommer Les nouveaux terrttoires urbains, Paris, Unesco/Maison des sciences de l'homme, 2001.

2. Jean GotTManN, Megalopolis. The Urbanized Northeastem Seabord of the Unted States, Cambridge (Massachusetts), Massachusercs Insticure of Technology Press, 1961.

3. François AsCHER, Mttapolis ou l'avenir des villes, Paris, Odile Jacob, 1995.

4. On assiste en France à l'émergence de la notion d'estuaire, terme qui doit sa nouvelle notoriété à la volonté politique de faire de ces places métropolitaines des espaces où vivre conciliant développenent économique et protection de l'environnement: l'estuaire de la Loire, entre Nantes et Saint-Nazaire, en constitue un exemple.

5. Pierre NORA, Lieux de memotre, Paris, Gallimard, 1984-1992. 
frontières, dans lequel l'identité de l'étranger et celle du territoire s'affrontent, et où l'identité migrante et l'identité urbaine ${ }^{6}$ évoluent dans une relation de convergence ou de divergence.

Au Québec, les recherches sur le territoire et sur les relations entre celui-ci et l'étranger sont désormais fondamentales et incontournables. Certains travaux, comme ceux de l'artiste René Derouin sur la culture du territoire ${ }^{7}$, ont ouvert des champs de réflexion originaux auxquels ont participé des géographes (dont Henri Dorion, Jean Morisset et Christian Morissonneau), des poètes (dont Claude Beausoleil, Nicole Brossard et Gaston Miron), des architectes et plusieurs artistes. D'autres approfondissent les domaines de la ville (dont Annick Germain, Pierre Delorme, Paul-André Linteau) ou du patrimoine (Lucie K. Morisset, Luc Noppen). Dans la recherche littéraire, on signalera deux champs d'investigation auxquels les chercheurs québécois ont grandement contribué: les relations de voyage (Pierre Berthiaume, Réal Ouellet, Normand Doiron) et les écritures migrantes.

Le présent numéro de Globe, que j'ai l'honneur de coordonner ${ }^{8}$, interroge les rapports entre l'étranger et le territoire au Québec. L'étranger est conçu en tant qu'univers des représentations et/ou sujet qui introduit une altérité sociale ou culturelle et qui intervient dans la configuration de la politique et dans le paysage social, culturel ou littéraire. Le territoire est quant à lui envisagé selon l'orientation adoptée par les sciences humaines ${ }^{9}$ (et particulièrement par la géographie) à la fin des années 1990, à savoir l'espace inscrit dans le social, le politique et le culturel, un espace susceptible d'être l'objet d'une appropriation et de devenir porteur d'identité, un espace qui est l'horizon de l'expérience humaine.

Les différentes approches qu'ont choisies les auteurs et auteures de ce dossier pour explorer les relations entre l'étranger et le territoire soulignent toutes le mouvement, la tension et les frontières. La métamorphose inhérente à la migration ou à la "migrance ${ }^{10}$ " chez le sujet immigrant reflète la

$$
+4
$$

6. Voir Carmen MATA BARREIRO, "Identité urbaine, identité migrante», Recherches sociographiques, vol. 45, $\mathrm{n}^{\circ} 1$, janvieravrl 2004, p. 39-58.

7. Voir René DEROujn [éd.], avec la collaboracion de Gilles LAPOINTE, Pour une culture du terrttotre, Montréal, L'Hexagone, 2001.

8. Je remercie Michel Lacroix et Karim Larose pour leurs suggescions et leurs remarques.

9. Pour une vision de synthèse, voir Jean-François STASZAK, "Espace», Sylvie MESURE et Patrick SAVIDAN [éd.], Dictionnaire des sciences bumatnes, Parıs, Presses universitaires de France, coll. «Quadrigen, 2006, p. 388-390

10. "J'aı forgé le mot migrance pour indiquer que la migracion est une douleur, une souffrance (la perte des racines, d'une certaine "naturalité") er, en même temps, une posture de distance, un lieu de vigilance." (Êmile OLIVIER, "Et me voilà otage et protagonisten, Les ecrits, no 95,1999, p. 171.) 
concurrence entre le pays quitté et le pays d'accueil, ainsi que la place de la mémoire et de l'héritage dans le choix de la langue et du territoire: le Québec est-il alors vécu et perçu comme un lieu d'exil ou comme un lieu de renaissance? Au sein de la société québécoise, comment se construisent les frontières entre, d'une part, l'ouverture à l'altérité et à la diversité et, d'autre part, la fidélité à une identité nationale, à un héritage culturel et à un projet de société ? Comment le flux migratoire fait-il évoluer la métropole montréalaise, un Montréal aux identités plurielles?

Parallèlement à l'étranger immigrant, l'étranger touriste crée un autre type d'interaction avec le territoire au Québec dans la définition du patrimoine. On peut ainsi se demander quel a été le poids de l'étranger dans l'imaginaire territorial québécois tel que constitué par la patrimonialisation.

Les articles qui composent ce numéro nous invitent à découvrir de nouveaux projets de recherche proches de l'axe thématique proposé et font écho à de nombreux débats intellectuels du Québec actuel. Ces chercheurs provenant de différentes disciplines créent des dialogues qui non seulement enrichissent notre connaissance du sujet, mais présentent des réflexions théoriques et méthodologiques dignes du plus haut intérêt.

Nous avons demandé à l'écrivain Naïm Kattan d'ouvrir ce numéro. Né à Bagdad et ayant immigré au Québec en 1954, cet auteur est romancier, nouvelliste, essayiste et critique littéraire. Dans l'avant-propos, il poursuit la réflexion, toujours intelligente et sensible, qu'il avait développée préalablement dans des livres tels que L'écrivain migrant. Essais sur des cités et des hommes ${ }^{11}$ et Les villes de naissance ${ }^{12}$. Il réfléchit ici à la façon dont l'étranger qui se déplace et change de territoire prend conscience de son état d'individu, et à comment il doit faire face à deux options, à savoir l'exil ou la proclamation d'une nouvelle naissance, ce deuxième choix comportant un nouveau départ, un recommencement social et humain. Dans cet engagement dans la quête d'un avenir, l'étranger ne peut pas se délester de son passé, de sa mémoire, de la "langue quittée", qui devient "sous-jacente" à la langue adoptée après un processus d'apprentissage humble et constant. Le texte de Naïm Kattan témoigne d'un vécu, d'une expérience de "passeur" de cultures et d' "écrivain du passage", et donne parallèlement à l'écrivain l'occasion de poursuivre une interrogation essentielle: "Serai-je partout un

$$
\div \div \div
$$

11. Naim KaTTAN, L'errivain migrant. Essaus sur des cites et des hommes, Montreal, Hurtubise HMH, 2001. 12. Naïm KaTtaN, Les villes de naissance, Montréal, Leméac, 2000. 


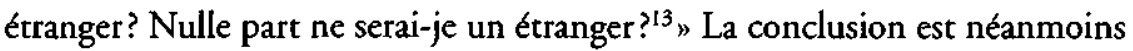
porteuse d'espoir : pour Kattan, accepter la métamorphose, vivre et réinventer une culture en mouvement, et traverser les frontières s'avèrent compatibles avec la volonté de garder sa mémoire et son héritage.

Le territoire, la mémoire et la langue sont les trois aspects qu'étudie Maude Labelle dans le roman de Ying Chen Les lettres chinoises. L'analyse de la façon dont prend forme l'entre-deux dans la construction textuelle du territoire et de la mémoire et dans l'utilisation de la langue est menée parallèlement à une réflexion, dans une perspective diachronique, qui détermine la critique de la catégorisation concernant l'écriture migrante.

C'est à partir de la philosophie politique que Stéphane Courtois aborde le nationalisme québécois, en répondant à une double question qui a été évoquée dans des débats publics : le nationalisme québécois est-il compatible avec le multiculturalisme "canadien"? Le nationalisme québécois et plus particulièrement ses politiques linguistiques sont-ils compatibles avec l'ouverture à la diversité culturelle et ethnique inhérente à une démocratie libérale? En vue de répondre à la première question, Stéphane Courtois mène une analyse comparée du nationalisme québécois et du multiculturalisme canadien afin d'en repérer les traits communs et d'identifier les éléments qui les opposent. L'ouverture à la diversité ethnique et culturelle et le respect des valeurs démocratiques fondamentales apparaissent comme étant partagés par les deux modèles: le Québec se présente comme une "société d'accueil» et une "société pluraliste». Ce qui ferait problème au Québec, selon l'auteur, c'est la vision de la nation canadienne proposée par Pierre Elliott Trudeau, dans laquelle le multiculturalisme est utilisé comme un instrument politique visant à occulter le multinationalisme. En ce qui concerne la deuxième question, celle de l'équilibre entre la préservation des voies garantissant le projet d'une "société distincte" et l'engagement en faveur du pluralisme au Québec, Courtois souligne les moyens mis en place pour bâtir cet équilibre: un "contrat moral" et une culture politique commune et publique, libérale, démocratique, pluraliste et entièrement ouverte à la diversité culturelle, qui s'avère aussi accueillante à l'endroit du multiculturalisme que le sont la plupart des démocraties libérales à travers le monde.

\section{$+4$}

13. Naïm KA'TTAN, "Avant-propos. Etranger et territorialité", Glabe. Revue tnternationale d'érudes québécoises, vol. 10, $\mathrm{n}^{\circ} 1,2007$, p. 35 . 
L'article de Lucie $\mathrm{K}$. Morisset s'intéresse quant à lui à la « mémoire patrimoniale des Québécois», et plus particulièrement à la constitution du patrimoine - la patrimonialisation - dans le Québec des années 1920-1930, au sein de la Commission des monuments historiques. Elle y montre comment le "touriste" provenant des provinces voisines et des États-Unis est perçu comme le destinataire prioritaire de cette patrimonialisation, en consommateur, en légataire et en interprète du patrimoine. Son analyse des Rapports de la Commission et des articles de La Presse permet d'observer comment la mise en valeur d'un «ici» territorialisé et spatialisé vise surtout à attirer le regard d'un Autre "touriste" et à raviver son estime envers le "Nous». Le Québec des monuments historiques devient ainsi un "ailleurs" aussi exotique pour les ressortissants que pour les Etats-Uniens. L'auteure explique également le lien entre l'étrange patrimonialisation, axée sur le patrimoine-souvenir, et la rumeur populaire voulant que l'Étranger, surtout Érats-Unien, rapporte «chez soi» les "trésors" du patrimoine québécois.

Pour leur part, les chercheures Annick Germain et Cécile Poirier proposent une réflexion sur l'évolution des configurations territoriales de l'immigration dans l'espace urbain montréalais et sur les défis que leur fluidité croissante pose aux chercheurs. L'article débute par une analyse diachronique des vagues migratoires et des concepts utilisés pour décrire les formes qu'elles ont prises dans la métropole ("petites patries", "quartiers ethniques", "quartiers multiethniques"). Après avoir décrit quatre grandes périodes de l'histoire de l'immigration à Montréal, qui aboutiront à la configuration de "quartiers multiethniques» au début des années 1990, les auteures constatent une diversification plus importante qui intervient dans le récent changement du paysage de l'immigration, sur l'île de Montréal et en banlieue. Cela les pousse à s'interroger sur la pertinence de proposer une "cinquième période». Leur analyse, rigoureusement fondée sur des enquêtes, prend la forme d'un essai et laisse percer, dans la dernière partie, une forte inquiétude suscitée par des indices qui signaleraient un changement du climat social et l'émergence d'attitudes xénophobes.

La problématique de l'" accommodement raisonnable ", qui apparaît en filigrane dans plusieurs articles de ce numéro, ainsi que le débat qui l'entoure, sont analysés par les sociologues Micheline Labelle et JeanClaude Icart, qui inaugurent ainsi une nouvelle section de la revue intitulée "Perspective», laquelle sera consacrée aux débats intellectuels du Québec actuel. Leur «lecture du débat sur les accommodements raisonnables» éclaire le problème, rend sa genèse intelligible et dissèque ses différentes composantes juridiques, sociales et politiques ainsi que ses dimensions 
éthique et discursive. Dans la première partie de l'article, les auteurs rappellent la définition du concept $d$ ' "accommodement raisonnable" qu'utilise la Commission des droits de la personne et des droits de la jeunesse du Québec (CDPDJ), en insistant sur le fait que l'accommodement raisonnable n'est ni une problématique d'immigration ni une problématique de multiculturalisme. En clarifiant ce concept et en constatant la façon dont le débat a dérapé, entraînant de graves conséquences sur le plan social et politique, ils nous font prendre conscience du degré de manipulation de l'information et de l'absence d'éthique chez certains médias et certains leaders politiques, qui s'engagent dans une voie proche du populisme. La deuxième partie s'intéresse à des enjeux sociétaux plus larges et à des facteurs internationaux et nationaux qui ont exercé une influence structurante dans le débat, dont la juridicisation du politique, la sécularisation de l'espace public, la question identitaire et le débat sur le nationalisme au Québec. Dans la conclusion, les auteurs soulignent que la délibération sur les accommodements raisonnables garde toute sa légitimité et qu'elle fournit l'occasion de revisiter les représentations de l'identité québécoise et de son devenir, à condition de savoir aller au-devant des écueils et des dangers, de bien gérer l'information et de promouvoir et garantir le respect de l'éthique.

La note critique qui accompagne le dossier, rédigée par Gilles Dupuis, porte sur la littérature migrante. L'auteur, après avoir évoqué l'intérêt que ce champ suscite, s'intéresse à deux livres parus récemment qui abordent la littérature migrante de façons très différentes: La littérature migrante dans l'espace francophone. Belgique - France - Québec - Suisse $e^{14}$, de Monique Lebrun et Luc Collès, avec la collaboration de Marie-Cécile Robinet, et Les passages obligés de l'écriture migrante ${ }^{15}$, de Simon Harel. Le premier, un ouvrage didactique qui propose un panorama de la littérature migrante dans l'espace francophone et des pistes d'exploitation pédagogique en classe de français, s'inscrit dans une démarche interculturelle. Simon Harel, quant à lui, poursuit dans son ouvrage une analyse de l'écriture migrante entreprise dans Le voleur de parcours. Identité et cosmopolitisme dans la littérature québécoise contemporaine $e^{16}$, en y ajoutant une réflexion originale sur le territoire.

$+4$

14. Monique Lebrun et Luc COLLES, avec la collaboration de Marie-Cécile RobineT, La litterature migrante dans l'espace francophone, Belgsque - France - Quebec - Sussse, Corti-Wodon, E.M.E. \& InterCommunications S.P.R.L., 2007. 15. Simon HAREL, Les passages obligés de l'écriture migrante, Montréal, XYZ, 2005.

16. Simon HAREL, Le voleur de parcours Identite et cosmopolitisme dans la littrature quebecoise contemporatne, Longueuil, Le Préambule, 1989. 


\section{D'UNE APPROCHE PLURIDISCIPLINAIRE \\ A UNE PERSPECTIVE TRANSDISCIPLINAIRE}

Penser le territoire ainsi que les relations entre l'étranger et le territoire exige de prendre la pleine mesure de leur diversité. Dans le milieu scientifique, actuellement, on remarque la volonté d'une "construction de savoirs" qui adopte la pluridisciplinarité comme approche fondamentale, un phénomène dont témoignent, entre autres, les titres de publications telles que Décrire la ville. La construction des savoirs urbains dans l'interaction et dans le texte ${ }^{17}$, de Lorenza Mondada, et La ville autrement ${ }^{18}$, de Pierre Delorme. Ce dernier expose d'ailleurs, dès l'incipit de son livre, son parti pris:

Comprendre la ville aujourd'hui suppose, peut-être plus que jamais, l'adoption d'une perspective pluridisciplinaire. En effet, la complexité des réalités urbaines de ce début de siècle exige que l'on envisage la ville sous de multiples aspects, parfois disparates en apparence mais, à l'analyse, toujours complémentaires ${ }^{19}$.

Cette perspective pluridisciplinaire qu'adopte et défend Delorme, et qui est aussi celle qui nourrit la trame et le mouvement du dossier présenté dans ce numéro, permet de saisir quelques aspects du complexe et vaste domaine de l'étranger et de la territorialité, et apporte un éclairage varié sur ses dimensions theorique et pratique.

Dans une revue telle que Globe, dont la mission est par définition pluridisciplinaire, il me semble important de réfléchir à certaines voies, à certains thèmes, concepts et initiatives qui permettent d'associer plus souvent la transdisciplinarité à la pluridisciplinarité. Ainsi, le dialogue entre les disciplines ne se produirait pas essentiellement dans l'espace de la réception, chez les lecteurs et lectrices, mais aussi dans l'espace et dans le processus de création et de recherche.

La transdisciplinarité est un concept plus ambitieux que la pluridisciplinarité: il dépasse l'idée d'addition ou de juxtaposition inhérente au préfixe "pluri" (du latin plures, "plusieurs"), et marque le passage ou le changement (le latin trans signifiant "par-delà»). L'approche d'un territoire comme la ville et l'étude de l'immigration ou de son expression littéraire,

$$
4+
$$

17. Lorenza MONDADA, Décrire la ville. La construction des savotrs urbains dans linteraction et dans le texte, Paris, Anthropos, coll. *Villes , 2000. Voir aussi son article « Paroles urbannes et figures de la ville. La polyphonie urbaine produit des ordres multiples de la ville", Urbanisme, $n^{\circ} 19,2003$, p. 8-15.

18. Pierre DELORME [éd.], La ville autrement, Sainte-Foy, Presses de l'Université du Québec, 2005.

19. Pierre Delorme, "Presentarion", Pierre Delorme [éd.], La ville autrement, op. cit., p. 1. 
qu'on la désigne par le terme "littérature migrante" ou par "écritures migrantes", devraient pouvoir intégrer le mouvement et le dialogue inhérents à cette notion, que Pierre Delorme partage pour l'analyse de la ville $e^{20}$.

Dans le domaine de l'analyse de l'immigration, Gérard Noiriel, historien français et membre du conseil scientifique de la Cité nationale de l'histoire de l'immigration, semble adopter cette voie. En effer, dans son dernier ouvrage, Immigration, antisémitisme et racisme en France (XIX $X X^{e}$ siècle). Discours publics, humiliations privées ${ }^{21}$, Noiriel mobilise les outils de la socio-histoire en les appliquant à un domaine qu'il n'avait jamais étudié auparavant, à savoir l'histoire du discours.

Or, l'approche transdisciplinaire nous semble aussi pertinente quand vient le temps de se tourner vers la littérature migrante ou les écritures migrantes au Québec. Les quelques considérations qui suivent ont pour but de mettre en évidence les concepts fédérateurs et des initiatives de dialogues entre la littérature et les sciences humaines et sociales.

\section{SCIENCES HUMAINES ET LITTÉRATURE. LES ÉCRITURES MIGRANTES}

Qu'elles viennent de la littérature ou des sciences humaines et sociales, plusieurs initiatives émergent et proposent de construire une complémentarité de deux regards. Pierre Bourdieu soulignait en 1992 que les sciences sociales devraient soumettre à examen le potentiel heuristique de la diégèse littéraire plutôt que de s'en défier ${ }^{22}$. Pour sa part, le sociologue Pierre Lassave affirmait plus récemment, dans Sciences sociales et littérature. Concurrence, complémentarité, interférences, que "la littérature constitue pour les sciences sociales, en tout ou en partie, un corpus de données, une ressource cognitive et un modèle d'énonciation ${ }^{23}$ ".

$+4+$

20. «La ville pose un problème épistémologique au chercheur qu tente d'en comprendre la nature et le fonctionnement. [...] L'érude de la ville constitue, pour les analystes, un modèle exemplaire de multidisciplinarité, voire de transdisciplinarité. " (Ibid, p. 9.)

21. Gérard NOIRIEL, Immigration, antisemttzme et racisme en France $\left(X I X^{\prime}-X X^{\prime}\right.$ siecle). Discours publics, humiliations proves, Paris, Librairie Arthème Fayard, 2007.

22. "Bref, je pense que la littérature, contre laquelle nombre de sociologues, dès l'origine et aujourd'hui encore, ont cru et croient devoir affirmer la scientificité de leur discipline [...], esr, en plus d'un point, en avance sur les sciences sociales et enferme tout un trésor de problèmes fondamentaux - concernant la théorie du récit, par exemple - que les sociologues devraient s'efforcer de reprendre à leur compte ec soumetrre à l'examen, au lieu de prendre ostentatoirement leurs distances avec des formes d'expression et de pensée qu'ils jugent comprometcantes. \# (Pierre BOURDIEU, Réponses. Pour une anthropologie reflextve, Paris, Seuil, 1992, p. 180.)

23. Pierre LASSAVE, Sciences sociales et ltztérarure. Concurrence, complementarite, interferences, Paris, Presses universitaires de France, 2002 , p. 37. 
Au Québec, on signalera le numéro de Recherches sociographiques consacré au rapport "Sciences sociales et littérature", dirigé par Micheline Cambron. Celle-ci rappelle, dans sa présentation ${ }^{24}$ du numéro, que le second colloque organisé par la revue, en 1964, s'intitulait "Littérature et société canadiennes-françaises" et que les termes de l'intitulé de la séance finale qualifiaient les relations entre la littérature et les sciences sociales et humaines de "conflictuelles et complémentaires». Quarante ans plus tard, Cambron s'interroge sur ces mêmes relations et conclut que, même si les sciences sociales, comme d'ailleurs les sciences humaines et surtout l'historiographie, semblent s'être considérablement éloignées de la littérature, il est possible de repérer divers ponts lancés ces dernières années entre les disciplines.

La ville, l'identité urbaine ${ }^{25}$, l'imaginaire urbain, l'imaginaire migrant constituent des lieux de rencontre, des concepts "dialogiques" pour l'étude desquels des perspectives transdisciplinaires s'avèrent nécessaires. La littérature migrante - dont les écritures migrantes au Québec -, qui véhicule et rend visibles les images et les imaginaires, les pratiques discursives, les représentations et les interprétations du territoire des acteurs migrants ainsi que leur processus d'apprivoisement et d'appropriation de ce territoire, tout autant que leur approche de la langue et de la mémoire, constitue un univers complexe et mouvant dont l'étude requiert une approche transdisciplinaire. Parallèlement, la nature hybride de la littérature migrante, où coexistent et interagissent, chez la plupart des écrivains et des écrivaines, création-fiction et réflexion, reflète leur analyse - souvent critique - des questions de l'immigration, de l'intégration, de l'ethnicisation du discours sur l'immigration et de la stigmatisation dont les immigrants sont victimes (dans certaines sociétés ou par certains acteurs de ces sociétés, tels que les médias). Cette nature hybride, donc, la rend apte à participer aux transformations de la société, non plus tellement à titre de reflet, mais bien à titre de discours structurant. Elle acquiert ainsi une dimension "performative ${ }^{26}$ ".

\section{IMAGINAIRE URBAIN, IMAGINAIRE MIGRANT}

Dans les recherches actuelles sur le territoire, sur la ville, une notion centrale s'impose : celle d'imaginaire. Des chercheurs tels que Pierre Delorme

$$
++4
$$

24. Micheline CAMBron, « Présentation", Recberches sociographiques, vol. XLIV, n 3, seprembre-décembre 2003, p. $429-431$.

25. Voir Carmen Mata Barreiro, op. cit.

26. Voir Marc Brosseau et Micheline Cambron, antre géographie et lictérature: frontières et perspecrives dialogiquess, Recherches sociographiques, vol. XI.IV, $n^{\circ} 3,2003$, p. 544. 
reconnaissent qu'il faut sortir de la sociologie urbaine habituelle et se tourner plutôt vers des notions qui permettent de saisir la complexité inhérente à la ville, telles que celle d'imaginaire, qu'il définit comme "une théorisation de l'être, singulier ou dans son espace collectif, qui donne un sens à sa vie en société27\%.

Certains chercheurs québécois provenant des sciences humaines et sociales, comme Luc Noppen et Lucie K. Morisset, historiens de l'architecture et chercheurs au Centre interuniversitaire d'études sur les lettres, les arts et les traditions (CÉLAT), ont par ailleurs montré, depuis des années, une attitude d'ouverture et de dialogue envers les chercheurs et chercheures qui travaillent sur l'imaginaire migrant, et particulièrement sur les relations entre l'imaginaire migrant et la ville dans les écritures migrantes. En témoignent les congrès qu'ils ont organisés et les ouvrages collectifs qu'ils ont dirigés, tels que Ville imaginaire/Ville identitaire. Échos de Québec ${ }^{28}$ ou Les identités urbaines. Échos de Montréal ${ }^{2}$.

\section{LA «MONTREALITÉ"}

Le concept de "montréalité», apparu récemment dans l'univers de la recherche, renferme des significations différentes et complémentaires. Dans le cadre de leurs recherches sur le patrimoine, et particulièrement sur les "images", les représentations et l'identité de la ville, Luc Noppen et Lucie K. Morisset font appel à la montréalité pour énoncer le caractère «identitaire" de Montréal:

La littérature s'est multipliée, récemment, qui a mis en évidence un imaginaire de particularité de la ville: Germain et Rose, Linteau, Manzagol et Bryant, Polèse, Tellier ont, parmi plusieurs autres et dans leurs différentes disciplines (de l'histoire à l'économie en passant par la géographie et la sociologie), fondé des analyses novatrices sur une spécificité rémanente. Comme, par exemple, Paris ou Boston (mais à sa manière), Montréal serait un "lieu spécial ", doté d'une identité distincte: c'est ce que nous avons nous-mêmes nommé tout

$$
++4
$$

27. Pierre Delorme, "De l'école de Chicago à l'imaginaire urbain», Pierre Delorme [éd.], La ville autrement, p. 24. 28. Lucie K. MORISSET, Luc NOPPEN et Denis SAINT-JACQUES [éd], Ville imaginaire/Ville identrtaire. Echos de Quebec, Québec, Nota bene, 1999. Voir dans cet ouvrage Carmen MATA BARREIRo, « Regard immıgrant sur la ville et voix immıgrances dans la ville: témoignages littéraires", p. 253-268.

29. Lucie K. MORISSET et Luc NOPPEN [éd.], Les identités urbatnes. Echos de Montréal, Québec, Nota bene, 2003. Voir dans cet ouvrage Carmen MATA BARREIRO, "Engagement et construction des identités urbaines dans la littérature francophone: la littérature mıgrante ex l'écriture au féminin *, p. 227-251. 
récemment, en reprenant le vocable utilisé par l'architecte Melvin Charney [...], la "montréalité" de Montréal ${ }^{30}$.

Chez Jocelyn Létourneau, le concept de montréalité se rapporte "à des écarts qualitatifs de plus en plus grands qui caractérisent Montréal par rapport à la "province" 31 ". Montréal apparaît comme "l'un des laboratoires d'interculturalité parmi les plus fascinants sur la planète ${ }^{32}$ ", "une zone où les passages identitaires sont intenses et nombreux entre les cultures formant ensemble - dans leurs consonances et leurs dissonances - la culture montréalaise ${ }^{33}$ ". La montréalité est ainsi définie comme

[une] sorte d'identité métropolitaine aux enracinements pluriethniques et aux résonances cosmopolites, [qui] infiltre tout le tissu social de la grande ville dont les solitudes précédentes, loin d'atteindre quelque plénitude, sont en proie aux vicissitudes caractéristiques des fins de règne ${ }^{34}$.

La lecture de la métropole, de l'évolution des configurations territoriales de l'immigration dans l'espace urbain montréalais, et de la montréalité est particulièrement riche chez certains écrivains migrants vivant ou ayant vécu à Montréal, comme Naïm Kattan, Émile Ollivier, Marco Micone, Mauricio Segura ou Marie-Célie Agnant. Ainsi, Mauricio Segura, dans son roman Côte-des-Nègres ${ }^{35}$, centre son regard sur la cohabitation des groupes ethnoculturels dans le quartier multiethnique de Côte-des-Neiges. Dans La brûlerie, un roman publié à titre posthume et que l'écrivain concevait comme une "écriture de la cartographie ${ }^{36}$ ", Émile Ollivier livre un témoignage de la diaspora haïtienne à Montréal, s'intéresse à la "géographie olfactive ${ }^{37}$ " de la ville et dresse un portrait sociologique du chemin de la Côte-des-Neiges.

$$
+4+
$$

30. Luc NOPPEN et Lucie K. MORISSET, "Ville et mort du patrimoine n, Pierre DELORME [éd.], La ville autrement, p. 51.52 .

31. Jocelyn Letourneau, «Postnationalisme? Rouvrir la quescion du Quebec», Cttés, n²3, aLe Québec, une autre Amérique. Dynamismes d'une identıtén, 2005, p. 18.

32. Ibrd., p. 19.

33. Ibrd

34. $\mathrm{lbs}$, p. 21.

35. Mauricio SEgura, Côte-des-Negres, Montréal, Boréal, 1998.

36. Émile OLıJier, La brâlerie, Montréal, Boréal, 2004, p. 7.

37. Marc BrosseaU, Des romans-geographes. Essah, Paris, L'Harmattan, 1996. Voir surtout le chapitre 4, p. 109-128. 


\section{PERSPECTIVE COMPARATISTE}

Est-ce que cette ouverture, ce dialogue entre les sciences humaines et sociales et la littérature qui se construit au Québec dans les écritures migrantes est aussi observable dans d'autres sociétés d'immigration, comme la société française? Est-ce que la littérature migrante y atteint une visibilité sociale et culturelle et un degré d'attention et d'intérêt en tant qu'objet de recherche et de formation universitaire comparables à ceux qu'on constate au Québec?

L'analyse de la situation dans d'autres pays d'immigration, notamment des pays européens ${ }^{38}$, révèle que le degré de visibilité et de reconnaissance de la littérature migrante y est bien inférieur à celui qu'on trouve au Québec. Les écritures migrantes au Québec, caractérisées par une grande richesse associée au dialogue et à la diversité de cultures que véhiculent les textes, ont été accueillies par des maisons d'édition importantes, ont désormais leur place dans les manuels scolaires et sont reconnues par les médias. Par ailleurs, des chercheurs tels que Pierre Nepveu, Simon Harel, Sherry Simon, Fulvio Caccia, Lucie Lequin et Maïr Verthuy, Pierre L'Hérault, Lise Gauvin et Daniel Chartier, parmi d'autres, ont étudié la littérature migrante et ont contribué à faire des écritures migrantes au Québec un objet d'étude modèle du point de vue scientifique et même éthique.

L'appartenance des écritures migrantes à la littérature québécoise est incontestable. Comme le rappelle Joël Des Rosiers, "ces écritures font bel et bien partie de notre patrimoine littéraire et culturel et marquent un tournant dans l'évolution de la littérature au Québec ${ }^{39}$ ". La construction de la catégorie "écritures migrantes" est le fruit d'un travail critique et de la reconnaissance de l'apport des écrivains migrants à la culture québécoise. L'existence de cette catégorie dans le paysage de la critique littéraire s'avère positive entre autres dans une perspective comparative: elle agit sur les représentations concernant les immigrants dans la société d'accueil en évitant l'association d'idées (immigrant $=$ travailleur étranger) et en valorisant leur apport, et elle permet de constituer un champ dans les milieux de la recherche, de la formation et de la promotion au niveau international, étant donné l'intérêt que ce corpus et ces écrivains suscitent ailleurs. Les chercheurs québécois n'ont pas bâti cette notion pour enfermer l'expression

$+\div$

38. Voir, par exemple. Carmen MATA BARREIRO, «La réception de la littérature migrance au Québec, dans l'Europe francophone et en Espagnen, Roberc LALIBERTE et Denis MONIERE [éd], Le Quebec au mirotr de l'Europe, Québec, Association internationale des études québtcoises, 2004, p. 89-98.

39. Joèl DES ROSIERS, "Mythologies mérisses ", Les écrits, n 95, avril 1999, p. 194. 
littéraire des écrivains ayant une identité culturelle et personnelle différente, et ils restent à l'écoute de l'actitude critique manifestée par certains écrivains migrants comme Régine Robin, Abla Farhoud ou Dany Laferrière. Dans le monde de la recherche littéraire québécoise, des études d'ensemble comme celle de Clément Moisan et Renate Hildebrand ${ }^{40}$ et des dictionnaires comme celui de Daniel Chartier ${ }^{41}$, cités dans la note critique de ce numéro, côtoient des monographies et des articles consacrés à l'œuvre d'écrivains comme Anne-Marie Alonzo, Naïm Kattan, Marie-Célie Agnant ou Aki Shimazaki, qui les étudient dans leur spécificité.

En France, on constate des progrès dans la visibilité des œuvres des écrivains migrants au cours des décennies 1990 et 2000. Les écrivains Tahar Ben Jelloun, Azouz Begag, Calixthe Beyala, Fatou Diome, Chahdortt Djavann et Shan Sa ont été publiés par des maisons d'édition importantes, et certaines de leurs œuvres ont été recommandées pour les classes de lycée (ainsi en est-il, par exemple, du roman La joueuse de $g 0^{42}$, de Shan Sa, qui a reçu le prix Goncourt des lycéens 2001). Mais, en ce qui concerne la reconnaissance de la littérature migrante ainsi que son adoption par l'institution littéraire française, beaucoup d'hésitations demeurent : des critères d'adoption fort arbitraires ont fait de Ionesco ou de Beckett des écrivains français, tandis que Tahar Ben Jelloun est présenté comme un auteur francophone. Et les catégories " littérature issue de l'immigration maghrébine" ou "littérature beur" posent des problèmes de classification, de désignation et de délimitation du corpus.

L'absence de consensus autour d'une catégorie qui intègre la migrance et "le passage [...] de l'identité assignée à celle de la traversée ${ }^{43}$ ", ainsi que les «nouvelles formes qui s'expérimentent: Destinerrance, Enracinerrance, Emigressence, migrance, transnational, transculturel, métissage ${ }^{44}$ " - lesquelles, selon Régine Robin, caractérisent les écritures migrantes au Québec - font que souvent la classification dans les bibliothèques françaises associe les auteurs migrants à leur pays ou à leur région géographique d'origine (par exemple, Calixthe Beyala au Cameroun ou à l'Afrique subsaharienne). Au cours des dernières années, toutefois,

$$
+4
$$

40. Clément MoIsan et Renate Hildobrand, Ces etrangers du dedans. Une histoire de l'écriture migrante au Quebec (1937-1997), Québec, Nota bene, 2001.

41. Daniel CHARTIER, Dictionnaire des écrivains émugrés au Québec, 1800-1999, Québec, Nota bene, 2003.

42. Shan SA, La jouewuse de go, Paris, Grasset, 2001.

43. Régine ROBIN, «Les champs littéraires sont-ils désespérément monolingues? Les écritures migrantes", Anne DE VAUCHER GRAVIU [éd.], D'autres rêves. Les tcrirures migrantes au Québec, Venise, Supernova, 2000, p. 35.

44. Ibsd., p. 40. 
plusieurs initiatives ont surgi pour "accueillir" la littérature migrante dans de nouvelles catégories ou par de nouvelles approches. Ainsi, Jacques Chevrier propose le néologisme "migritude ${ }^{45}$ " pour loger la nouvelle génération d'écrivains africains, qui ont délaissé Dakar ou Douala au profit de Paris, Caen ou Pantin. Jean-Marc Moura amorce quant à lui un renouvellement théorique à la lumière de la théorie postcoloniale qui insisterait plutôt sur les formes textuelles de l'hybridité et qui, dans son approche de la «littérature de l'immigration maghrébine",

placerait [...] l'écriture de ces auteurs, d'Azouz Begag à Faridah Belghoul, au couur d'un débat actuel (ec non tranché) entre discours d'assimilation à la nation et discours d'une mémoire revendiquée par les enfants d'immigrants: débat sur une citoyenneté pleine mais qui passerait aussi par le contact permanent avec la culture d'origine. Il s'agirait d'étudier l'écriture d'une identité en formation fondée sur une négociation culturelle constante ${ }^{46}$.

En ce qui concerne les mouvements transdisciplinaires, on perçoit certains progrès, même si ceux-ci restent timides. Ainsi, dans le domaine historiographique, deux excellents titres parus en 2007 traitent de l'immigration en France, tout en affichant des attitudes différentes dans leur dialogue avec les écrivains migrants. D'une part, le livre Immigrances. L'immigration en France au $X X^{*}$ siècle ${ }^{47}$, dirigé par Benjamin Stora et Émile Témime, consacre un chapitre à «L'immigré dans la fiction, des années soixante à nos jours", incluant le cinéma et la littérature. D'autre part, l'ouvrage de Gérard Noiriel Immigration, antisémitisme et racisme en France $\left(X I X^{e}-X X^{*}\right.$ siècle). Discours publics, humiliations privées ${ }^{48}$, déjà cité dans cette introduction, est remarquable par la profondeur de son analyse et sa solide attitude éthique - qui rejoint les préoccupations de certains auteurs de ce numéro de Globe par rapport au danger inhérent à l'ethnicisation du discours sur l'immigration et à la stigmatisation (dont «le retour de la "racaille" et les nouveaux habits du discours national-sécuritaire ${ }^{49} n$ ) -, mais évoque trop

$$
++4
$$

\footnotetext{
45. Jacques CHEVRIER, "Afrique(s)-sur-Seine. Autour de la notion de "migritude" ", Notre Libratre. Renue des littetratures du Sud, no 155-156, juillet-décembre 2004, p. 96-100.

46. Jean-Marc MOURA, Litteratures francophones et theonte postcoloniale, Paris, Presses universitares de France, 1999 , p. 155. Voir aussi Boniface MONGO-MBOUSSA, "Jean-Marc Moura. LAilleurs", Destr d'Afrique, Paris, Gallımard, coll. "Continents noirs", 2002, p. 291-303.

47. Benjamin STORA et Émile TÉMIME [éd.], Immigrances. L'immigration en France au $X X^{\prime}$ stècle, Paris, Hachetre Lırtératures, 2007.

48. Gérard NoIRIEL, op. cit.

49. Ibtd, p. 665-667.
} 
brièvement des écrivains comme Chahdortt Djavann ou Azouz Begag et ne leur accorde pas la parole comme à de véritables interlocuteurs.

Ce numéro de Globe vous convie ainsi, chers lecteurs, chères lectrices, à explorer de nouveaux territoires, à vous plonger dans des débats sociaux passionnants, à réfléchir sur des sujets tels que la mémoire, la langue et l'identité, associés au territoire et à l'étranger, et enfin à franchir des frontières tant spatiales que disciplinaires. 